\title{
The Association of Small Dense Low-Density Lipoprotein Cholesterol and Coronary Heart Disease in Subjects at High Cardiovascular Risk
}

\author{
Mayu Higashioka ${ }^{1,2}$, Satoko Sakata ${ }^{1,3,4}$, Takanori Honda ${ }^{1}$, Jun Hata 1,3, 4, Mao Shibata ${ }^{1,3}$, Daigo Yoshida ${ }^{1}$, \\ Kenichi Goto ${ }^{4}$, Takanari Kitazono ${ }^{3,4}$, Haruhiko Osawa ${ }^{2}$ and Toshiharu Ninomiya ${ }^{1,3}$ \\ ${ }^{1}$ Department of Epidemiology and Public Health, Graduate School of Medical Sciences, Kyushu University, Fukuoka, Japan. \\ ${ }^{2}$ Department of Diabetes and Molecular Genetics, Graduate School of Medicine, Ehime, Japan. \\ ${ }^{3}$ Center for Cohort Studies, Graduate School of Medical Sciences, Kyushu University, Fukuoka, Japan. \\ ${ }^{4}$ Department of Medicine and Clinical Science, Graduate School of Medical Sciences, Kyushu University, Fukuoka, Japan.
}

Aim: The present study aims to investigate the association between serum small dense low-density lipoprotein (sdLDL) cholesterol level and the development of coronary heart disease (CHD) in subjects at high cardiovascular risk.

Methods: A total of 3,080 participants without prior cardiovascular disease (CVD), aged $\geq 40$ years, were followed up for a median of 8.3 years, which were divided into two groups, those with serum sdLDL cholesterol levels of $<35 \mathrm{mg} / \mathrm{dL}$ or $\geq 35 \mathrm{mg} / \mathrm{dL}$. Then, subjects were stratified by the status of diabetes, CVD-related comorbidities (defined as the presence of diabetes, chronic kidney disease, or peripheral artery disease), and the CVD risk assessment according to the Japan Atherosclerosis Society Guidelines. The hazard ratios (HRs) and 95\% confidence intervals (CIs) were computed using a Cox proportional hazards model.

Results: During the follow-up, 79 subjects developed CHD. The risk for incident CHD was higher in subjects with serum sdLDL cholesterol of $\geq 35 \mathrm{mg} / \mathrm{dL}$ than those with sdLDL cholesterol of $<35 \mathrm{mg} / \mathrm{dL}$ (HR 2.09, 95\%CI 1.26-3.45) after adjusting for traditional risk factors. In the subgroup analyses, the multivariableadjusted HR for incident CHD increased significantly in those with serum sdLDL cholesterol of $\geq 35 \mathrm{mg} / \mathrm{dL}$ among subjects with diabetes (HR 2.76, 95\%CI 1.09-7.01), subjects with CVD-related comorbidities (HR 2.60, 95\%CI 1.21-5.58), and high-risk category defined as the presence of CVD-related comorbidities or a Suita score of $\geq 56$ points (HR $1.93,95 \%$ CI $1.02-3.65$ ).

Conclusions: Elevated serum sdLDL cholesterol was associated with the development of CHD even in subjects at high cardiovascular risk.

Key words: Small dense LDL cholesterol, Coronary heart disease, Risk assessment, Prospective study, High-risk population

Abbreviations: BMI, body mass index; CHD, coronary heart disease; CI, confidence interval; CVD, cardiovascular disease; ECG, electrocardiogram; eGFR, estimated glomerular filtration rate; HbAlc, hemoglobin A1c; HDL, high-density lipoprotein; HR, hazard ratio; LDL, low-density lipoprotein; MESA, Multi-Ethnic Study of Atherosclerosis; sdLDL, small dense low-density lipoprotein.

\section{Introduction}

Coronary heart disease (CHD) is a common noncommunicable disease and one of the leading causes of mortality worldwide. According to a report from the Global Burden of Diseases study, in 2017, there were 8.93 million deaths from CHD, and CHD was the leading cause of years of life lost ${ }^{1)}$. In Japan,

Address for correspondence: Satoko Sakata, Department of Epidemiology and Public Health, Graduate School of Medical Sciences, Kyushu University 3-1-1 Maidashi, Higashi-ku, Fukuoka City, Fukuoka, 812-8582, Japan E-mail: ssakata@intmed2.med.kyushu-u.ac.jp

Received: January 22, 2020 Accepted for publication: March 4, 2020

Copyright@2021 Japan Atherosclerosis Society

This article is distributed under the terms of the latest version of CC BY-NC-SA defined by the Creative Commons Attribution License. 
heart disease is the second leading cause of death in 2017, with CHD accounting for over $30 \%$ of heart disease deaths ${ }^{2}$. Hence, preventing the development of CHD is a highly important issue in contemporary and future public health on a global scale.

Previous observational studies and clinical trials have revealed that serum low-density lipoprotein (LDL) cholesterol is a significant risk factor for the development of $\mathrm{CHD}^{3)}$. The Guidelines for the Prevention of Atherosclerotic Cardiovascular Disease 2017 from the Japan Atherosclerosis Society recommend monitoring and intervention on LDL cholesterol for primary prevention of atherosclerotic cardiovascular disease (CVD), of which target levels of LDL cholesterol-lowering treatment are categorized according to the absolute risk of CHD estimated by the Suita score, a risk index used to estimate the 10-year risk of CHD for the Japanese population ${ }^{4-5}$.

Recently, the difference in the atherogenic effect across differently sized LDL particles has attracted attention. In particular, small dense LDL (sdLDL), which is small in size and highly dense, has been shown to have more atherogenic effect than other LDL particles ${ }^{6-8)}$. Several prospective cohort studies have reported the significant association between serum sdLDL cholesterol and the development of CHD regardless of serum LDL cholesterol levels ${ }^{9-11)}$. Our study group also reported a significant association between serum sdLDL cholesterol and the development of CHD in a general Japanese population; the cutoff value of sdLDL cholesterol for discriminating the risk of developing CHD was around $35 \mathrm{mg} / \mathrm{dL}^{12}$. However, it is still unknown whether the association between sdLDL cholesterol and CHD is observed even in individuals at high cardiovascular risk. It is of particular importance to investigate the association in subpopulations with varied cardiovascular risks to evaluate clinical usefulness of measuring sdLDL in routine care settings.

\section{Aim}

The present study aims to clarify the association between sdLDL cholesterol and CHD among Japanese general population stratified by predefined CHD risks using the risk stratification algorithm of the Japan Atherosclerosis Society 2017 Guidelines.

\section{Methods}

Study Population

A detailed description of the Hisayama study was published previously ${ }^{13}$. Briefly, a total of 3,384 residents aged 40 years or older $(78.2 \%$ of the total popu- lation of this age group) underwent the screening survey for the present study in 2007 and 2008. After excluding 8 subjects who did not consent to participate in the study, 223 subjects who had past history of stroke or CHD, and 73 subjects for whom serum sdLDL cholesterol data were lacking, the remaining 3,080 subjects $(1,290$ men and 1,790 women) were enrolled in the present study.

The study was approved by the Kyushu University Institutional Review Board for Clinical Research, and written informed consent was obtained from all participants.

\section{Follow-Up Survey}

The subjects were followed up prospectively until November 2015 or their death (median, 8.3 years) by annual health examinations or by mail or telephone for any subject who did not undergo the examination or who moved out of the town as described previously ${ }^{14)}$. The development of CHD was also checked by a daily monitoring system organized by the study team, local physicians, and members of the Health and Welfare Office of the town. Subjects with suspected CHD events were evaluated on the basis of all available clinical information, and their diagnoses were adjudicated by study team physicians. When a subject died, an autopsy was performed at the Department of Pathology of Kyushu University, if consent for autopsy was obtained. During the follow-up period, autopsy examination was performed for 192 $(57.8 \%)$ of 332 deceased subjects. In addition to the deceased cases, four subjects were lost to follow up, all of whom were subjects who moved out of the town.

\section{Small Dense Low-Density Lipoprotein Cholesterol Measurement}

At the screening examination, portions of the plasma specimens were stored at $-80^{\circ} \mathrm{C}$ until serum sdLDL cholesterol concentrations were measured in 2014 on a Hitachi 7180 automated chemistry analyzer using a homogeneous assay (sdLDL-EX "SEIKEN"; Denka Seiken, Tokyo) ${ }^{15}$. The subjects were divided into two groups, those with serum sdLDL cholesterol of $<35$ and of $\geq 35 \mathrm{mg} / \mathrm{dL}$, which is the cutoff value reported previously ${ }^{12)}$.

\section{Outcomes}

The primary outcome of the present study was CHD. The criteria for the diagnosis of CHD included first-ever fatal and nonfatal myocardial infarction, silent myocardial infarction, sudden cardiac death within 1 hour after the onset of acute illness, coronary angioplasty, and bypass grafting. The diagnosis of myocardial infarction was based on detailed clinical 
information and at least two of the following findings: typical clinical symptoms, electrocardiogram (ECG) evidence of myocardial infarction, elevated cardiac enzymes, or morphologic findings, including echocardiographic, scintigraphic, or angiographic abnormalities compatible with myocardial injury, or myocardial necrosis or scars more than $1 \mathrm{~cm}$ in diameter at autopsy $^{14,16)}$.

\section{Other Variables}

Information on medical history including the presence of peripheral artery disease; medication for hypertension, diabetes, and dyslipidemia; smoking habits; alcohol intake; and physical activity at baseline was obtained using a standard self-administered questionnaire and was checked by trained interviewers. Smoking and drinking habits were classified as either current use or not. Current smoking was defined as smoking at least one cigarette per day. Current drinking was defined as drinking at least one alcoholic beverage per month. The subjects engaging in sports or other forms of exertion $\geq 3$ times a week during their leisure time made up a regular exercise group. Body height and weight were measured with the subjects in light clothing without shoes, and body mass index (BMI) was calculated $\left(\mathrm{kg} / \mathrm{m}^{2}\right)$. Blood pressure was measured three times in a sitting position using an automated sphygmomanometer (BP-203 RVIIIB; Omron Healthcare), and the mean of three measurements was used in the present analysis. Hypertension was defined as blood pressure $\geq 140 / 90 \mathrm{mmHg}$ and/ or current use of antihypertensive agents. ECG abnormalities were defined as left ventricular hypertrophy (Minnesota code 3-1), ST depression (4-1, 4-2, or 4-3), or atrial fibrillation/flutter (8-3). Blood samples were collected from an antecubital vein. Plasma glucose levels were measured by the hexokinase method. Diabetes mellitus was defined as a fasting plasma glucose level $\geq 126 \mathrm{mg} / \mathrm{dL}, 2$-hour post load or casual glucose level of $\geq 200 \mathrm{mg} / \mathrm{dL}$, or current use of glucose-lowering agents (i.e., oral glucose-lowering agents or insulin). Prediabetes was defined as a fasting plasma glucose level of 110 to $125 \mathrm{mg} / \mathrm{dL}$ and 2-hour post load glucose level of $<200 \mathrm{mg} / \mathrm{dL}$ or fasting plasma glucose level of $<126 \mathrm{mg} / \mathrm{dL}$ and 2-hour post load glucose level of 140 to $199 \mathrm{mg} / \mathrm{dL}$. Hemoglobin A1c (HbA1c) was measured by latex aggregation immunoassay using a Determiner HbA1c kit (Kyowa Medex, Tokyo). Serum LDL cholesterol and high-density lipoprotein (HDL) cholesterol were measured enzymatically, and serum triglycerides were measured with colorimetric method. The estimated glomerular filtration rate (eGFR) was calculated using the Chronic Kidney Disease Epidemiology Collaboration equation with a Japanese coefficient of 0.813 , for which serum creatinine was measured enzymatically ${ }^{17)}$. Chronic kidney disease was defined as eGFR $<60 \mathrm{~mL} /$ $\mathrm{min} / 1.73 \mathrm{~m}^{2}$ or proteinuria ( $\geq 1+$ on dipstick) according to the Kidney Disease Improving Global Outcomes guideline ${ }^{18)}$.

\section{Risk Assessments for Cardiovascular Disease}

We performed the risk assessment for CVD according to the Guidelines for Prevention of Atherosclerotic Cardiovascular Disease 2017 from the Japan Atherosclerosis Society ${ }^{4)}$ (Supplementary Fig.1). CVD-related comorbidities were defined as the presence of diabetes, chronic kidney disease, and/or peripheral artery disease. First, subjects with CVDrelated comorbidities were classified as a high-risk category. Next, the Suita scores were calculated with the variables of age, sex, smoking habits, blood pressure level, serum LDL and HDL cholesterol levels, and prediabetes among subjects without CVD-related comorbidities. Because data on family history of premature CHD were not available, 0 point was assigned to all participants. Subjects were classified into categories based on the total Suita score as follows: low-risk category ( $\leq 40$ points), moderate-risk category (41-55 points), and high-risk category ( $\geq 56$ points $)^{4)}$.

\section{Statistical Analysis}

The significance of differences in the mean values or frequencies of risk factors between subjects with sdLDL cholesterol of $<35 \mathrm{mg} / \mathrm{dL}$ and those with sdLDL cholesterol of $\geq 35 \mathrm{mg} / \mathrm{dL}$ was tested using Student's $t$ test or the $\chi^{2}$ test, respectively. Serum triglyceride level was shown as median and interquartile range in the baseline characteristics, because of the skewed distribution. The incidence rate of CHD was calculated using the person-year method. The Cox proportional hazards model was used to estimate the hazard ratio (HR) and its 95\% confidence intervals (CIs) for the development of CHD by subpopulations stratified by the status of (1) diabetes, (2) the CVDrelated comorbidities, and (3) the CVD risk assessment. In the multivariable analysis, the risk estimates were adjusted for potential confounding factors at baseline-namely, age, sex, systolic blood pressure, use of antihypertensive agents, HbA1c, use of glucoselowering agents, serum HDL cholesterol, lipid-modifying agents, BMI, eGFR, ECG abnormalities, current smoking, current drinking, and regular exercise. The heterogeneities in the association between subgroups were tested by adding multiplicative interaction terms to the relevant Cox model. A two-sided value of $p<$ 0.05 was considered statistically significant in all analyses. Statistical analyses were conducted using Statisti- 
Table 1. Baseline characteristics of overall subjects and characteristics according to small dense LDL cholesterol level.

\begin{tabular}{|c|c|c|c|c|}
\hline \multirow{2}{*}{ Variables } & \multirow{2}{*}{ Overall subjects } & \multicolumn{2}{|c|}{ Serum sdLDL cholesterol level } & \multirow{2}{*}{$p$ value $^{\mathrm{a}}$} \\
\hline & & $<35 \mathrm{mg} / \mathrm{dL}$ & $\geq 35 \mathrm{mg} / \mathrm{dL}$ & \\
\hline$N$ & 3,080 & 1,728 & 1,352 & - \\
\hline Age (years) & $63(13)$ & $64(14)$ & $62(11)$ & $<0.001$ \\
\hline Men $(\%)$ & 41.9 & 36.1 & 49.3 & $<0.001$ \\
\hline Systolic blood pressure (mmHg) & $132(19)$ & $129(20)$ & $136(18)$ & $<0.001$ \\
\hline Use of antihypertensive agents (\%) & 29.5 & 29.4 & 29.7 & 0.87 \\
\hline Diabetes (\%) & 15.0 & 11.3 & 19.7 & $<0.001$ \\
\hline Prediabetes (\%) & 23.1 & 18.5 & 29.1 & $<0.001$ \\
\hline HbAlc (\%) & $5.5(0.8)$ & $5.4(0.7)$ & $5.6(0.8)$ & $<0.001$ \\
\hline Use of glucose-lowering agents (\%) & 6.5 & 6.0 & 7.1 & 0.23 \\
\hline Serum LDL cholesterol (mg/dL) & $122(32)$ & $107(24)$ & $141(30)$ & $<0.001$ \\
\hline Serum non-HDL cholesterol (mg/dL) & $142(36)$ & $123(26)$ & $167(31)$ & $<0.001$ \\
\hline Serum HDL cholesterol (mg/dL) & $67(18)$ & $72(18)$ & $61(16)$ & $<0.001$ \\
\hline Serum triglycerides $(\mathrm{mg} / \mathrm{dL})^{\mathrm{b}}$ & $100(73-143)$ & $82(62-107)$ & $136(101-192)$ & $<0.001$ \\
\hline Use of lipid-modifying agents (\%) & 13.9 & 14.7 & 12.9 & 0.14 \\
\hline BMI $\left(\mathrm{kg} / \mathrm{m}^{2}\right)$ & $23(4)$ & $22(3)$ & $24(3)$ & 0.68 \\
\hline $\mathrm{eGFR}\left(\mathrm{ml} / \mathrm{min} / 1.73 \mathrm{~m}^{2}\right)$ & $75(13)$ & $75(13)$ & $76(12)$ & $<0.001$ \\
\hline Urinary protein $(\%)$ & 5.9 & 5.2 & 6.9 & 0.046 \\
\hline Chronic kidney disease (\%) & 13.7 & 14.0 & 13.5 & 0.71 \\
\hline Electrocardiogram abnormalities (\%) & 15.3 & 14.2 & 16.8 & 0.046 \\
\hline History of peripheral artery disease & 0.2 & 0.3 & 0.2 & 0.41 \\
\hline Current smoking (\%) & 19.9 & 16.6 & 24.3 & $<0.001$ \\
\hline Current drinking (\%) & 47.6 & 42.8 & 53.9 & $<0.001$ \\
\hline Regular exercise (\%) & 11.9 & 11.8 & 11.9 & 0.94 \\
\hline
\end{tabular}

Abbreviations: BMI, body mass index; eGFR, estimated glomerular filtration rate; HbA1c, hemoglobin A1c; HDL, high-density lipoprotein; LDL, low-density lipoprotein; sdLDL, small dense low-density lipoprotein.

Data are presented as the mean values (standard deviation), percentages, or median (interquartile range).

a The significance of differences in mean values and frequencies of risk factors between the level of sdLDL cholesterol were tested by Student's $t$ test and chi-square test, respectively.

${ }^{\mathrm{b}}$ Only subjects with available fasting blood samples were included in the analysis using serum triglyceride $(n=3,056)$.

cal Analysis Software version 9.4 (SAS Institute, Cary, NC).

\section{Results}

The baseline characteristics of the total study population and those with serum sdLDL cholesterol levels of less than $35 \mathrm{mg} / \mathrm{dL}$ and of $35 \mathrm{mg} / \mathrm{dL}$ or more are shown in Table 1 . The mean values of systolic blood pressure, HbA1c, serum LDL cholesterol, serum non-HDL cholesterol, and eGFR; the median value of serum triglycerides; and the frequencies of male sex, diabetes, prediabetes, urinary protein, ECG abnormalities, current smoking, and current drinking were significantly higher in subjects with serum sdLDL cholesterol levels of $\geq 35 \mathrm{mg} / \mathrm{dL}$ compared to serum sdLDL cholesterol levels of $<35 \mathrm{mg} / \mathrm{dL}$, whereas the mean values of age and serum HDL cholesterol were significantly lower.
During the follow-up period, 79 subjects experienced a first-ever CHD event. The crude cumulative incidence of CHD was significantly higher in subjects with serum sdLDL cholesterol levels of $\geq 35 \mathrm{mg} / \mathrm{dL}$ than $<35 \mathrm{mg} / \mathrm{dL}$ (log-rank $p<0.001$, Fig. 1). Table 2 demonstrates the age- and sex-adjusted and multivariable-adjusted HRs (95\%CIs) of serum sdLDL cholesterol of $\geq 35 \mathrm{mg} / \mathrm{dL}$ against $<35 \mathrm{mg} / \mathrm{dL}$ for the development of CHD, in overall subjects and in the subgroups according to diabetic status, the presence or absence of CVD-related comorbidities (diabetes, chronic kidney disease, and/or peripheral artery disease), and the risk assessment status based on the guidelines from the Japan Atherosclerosis Society. In overall subjects, the age- and sex-adjusted HR for the development of CHD was 2.44 (95\%CI, 1.52-3.90) for serum sdLDL cholesterol of $\geq 35 \mathrm{mg} / \mathrm{dL}$ as compared with serum sdLDL cholesterol of $<35 \mathrm{mg} / \mathrm{dL}$. This association remained significant even after adjust- 


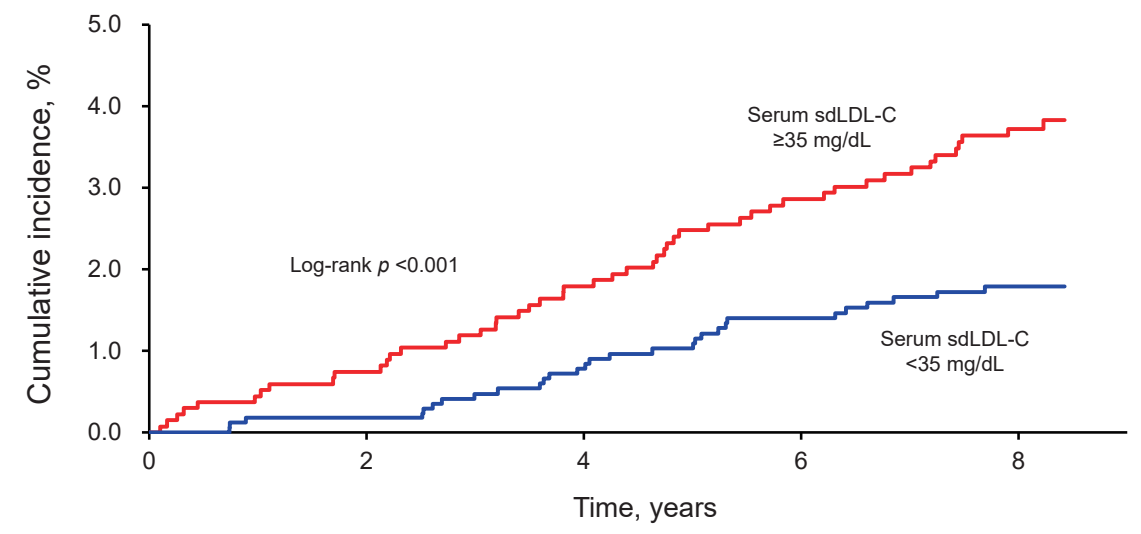

\begin{tabular}{|c|c|c|}
\hline & $\begin{array}{c}\text { sdLDL-C }<35.0 \mathrm{mg} / \mathrm{dL} \\
\mathrm{n}=1728\end{array}$ & $\begin{array}{c}\text { sdLDL-C } \geq 35.0 \mathrm{mg} / \mathrm{dL} \\
\mathrm{n}=1352\end{array}$ \\
\hline Number of events/person year & $29 / 13325$ & $50 / 10692$ \\
Incidence rate per 1,000 person year & 2.18 & 4.68 \\
$(95 \% \mathrm{Cl})$ & $(1.51-3.13)$ & $(3.55-6.16)$ \\
\hline
\end{tabular}

Fig. 1. Crude cumulative incidence of coronary heart disease according to serum small dense low-density lipoprotein cholesterol

Abbreviations: sdLDL-C, small dense low-density lipoprotein cholesterol.

ment for age, sex, systolic blood pressure, use of antihypertensive agents, $\mathrm{HbA1c}$, use of glucose-lowering agents, BMI, eGFR, ECG abnormalities, current smoking, current drinking, and regular exercise (HR 2.09, 95\%CI 1.26-3.45). In the subgroup analysis, the multivariable-adjusted HRs for the development of CHD increased significantly in those with serum sdLDL cholesterol of $\geq 35 \mathrm{mg} / \mathrm{dL}$ compared to those with serum sdLDL cholesterol of $<35 \mathrm{mg} / \mathrm{dL}$ in both subjects with and without diabetes (without diabetes: HR 1.89, 95\%CI 1.01-3.52; with diabetes: HR 2.76, 95\%CI 1.09-7.01), in subjects with CVD-related comorbidities (HR 2.60, 95\%CI 1.21-5.58), and in subjects in the high-risk category (HR 1.93, 95\%CI 1.02-3.65). There was no evidence of significant heterogeneities in the association between these subgroups (all $p$ for heterogeneity $>0.5$ ). We also divided subjects into low-risk category and moderate-/highrisk category for the risk assessment status, and the multivariable-adjusted $\mathrm{HR}$ of the development of CHD was 5.77 (95\%CI 0.25-133.23) among subjects in the low-risk category and 1.70 (95\%CI $1.05-$ 2.76) among subjects in the moderate-/high-risk category, without any evidence of heterogeneity between subgroups ( $p$ for heterogeneity $=0.77$ ). When we focus on the results of the subjects in the high-risk category for the risk assessment status, the age- and sexadjusted cumulative incidence of CHD was higher in subjects with serum sdLDL cholesterol levels of $\geq 35$ $\mathrm{mg} / \mathrm{dL}$ than those with $<35 \mathrm{mg} / \mathrm{dL}(p=0.03$, Supplementary Fig. 2).
Fig. 2 shows the joint influence of serum sdLDL cholesterol levels and the risk assessment status on the development for CHD. As compared to subjects with serum sdLDL cholesterol of $<35 \mathrm{mg} / \mathrm{dL}$ and at low-/ moderate-risk category, the multivariable-adjusted HRs for the development of CHD increased significantly in subjects with serum sdLDL cholesterol of $<$ $35 \mathrm{mg} / \mathrm{dL}$ and at high-risk category (HR 2.67, 95\%CI 1.26-5.66) or in subjects with serum sdLDL cholesterol of $\geq 35 \mathrm{mg} / \mathrm{dL}$ and at low-/moderate-risk category (HR 2.05, 95\%CI 1.01-4.16). Moreover, subjects with serum sdLDL cholesterol of $\geq 35 \mathrm{mg} / \mathrm{dL}$ and in the high-risk category had an additive excess risk of CHD events (HR 5.13, 95\%CI 2.64-9.98). Similar joint excess risks of CHD events were observed according to the presence or absence of diabetes or CVD-related comorbidities (Supplementary Fig. 3).

Finally, we examined the influence of serum sdLDL cholesterol on the development of CHD between subgroups of other lipid parameters-namely, serum LDL cholesterol, serum non-HDL cholesterol, serum HDL cholesterol, and serum triglycerides-in subjects in the high-risk category. The correlation between serum sdLDL cholesterol and other lipid parameters in those subjects were relatively strong (Supplementary Table 1). The multivariable-adjusted risk of CHD tended to be higher in subjects with serum sdLDL cholesterol of $\geq 35 \mathrm{mg} / \mathrm{dL}$ than in those with serum sdLDL cholesterol of $<35 \mathrm{mg} / \mathrm{dL}$ regardless of whether the levels of the other lipid parameters were higher or lower, although the HRs did not reach 
Table 2. Hazard ratios for the development of coronary heart disease according to serum small dense low-density lipoprotein cholesterol levels in subgroups of the status of cardiovascular risk

\begin{tabular}{|c|c|c|c|c|c|c|}
\hline & \multirow{2}{*}{\multicolumn{2}{|c|}{$\begin{array}{c}\begin{array}{c}\text { No. of } \\
\text { events/subjects }\end{array} \\
\text { Serum sdLDL-C }(\mathrm{mg} / \mathrm{dL})\end{array}$}} & \multirow{2}{*}{\multicolumn{2}{|c|}{$\begin{array}{c}\begin{array}{c}\text { Crude incidence rate } \\
\text { (per 1,000 person-years) }\end{array} \\
\text { Serum sdLDL-C }(\mathrm{mg} / \mathrm{dL})\end{array}$}} & \multicolumn{2}{|c|}{$\begin{array}{c}\text { HR }(95 \% \mathrm{CI}) \text { of } \\
\text { serum sdLDL-C } \geq 35 \mathrm{mg} / \mathrm{dL} \text { vs. }<35 \mathrm{mg} / \mathrm{dL}\end{array}$} \\
\hline & & & & & \multirow{2}{*}{$\begin{array}{l}\text { Age- and } \\
\text { sex-adjusted }\end{array}$} & \multirow{2}{*}{$\begin{array}{l}\text { Multivariable- } \\
\text { adjusted }\end{array}$} \\
\hline & $<35$ & $\geq 35$ & $<35$ & $\geq 35$ & & \\
\hline Overall subjects $^{\mathrm{a}}$ & $29 / 1728$ & $50 / 1352$ & 2.18 & 4.68 & $2.44(1.52-3.90)$ & $2.09(1.26-3.45)$ \\
\hline \multicolumn{7}{|l|}{ Diabetes $^{b}$} \\
\hline Presence & $8 / 195$ & $21 / 266$ & 5.43 & 10.32 & $2.30(0.999-5.29)$ & $2.76(1.09-7.01)$ \\
\hline$p$ for heterogeneity & & & & & 0.81 & 0.87 \\
\hline \multicolumn{7}{|l|}{ CVD-related comorbidities ${ }^{c}$} \\
\hline Absence & $17 / 1340$ & $25 / 967$ & 1.61 & 3.22 & $2.35(1.25-4.43)$ & $1.86(0.94-3.69)$ \\
\hline Presence & $12 / 388$ & $25 / 385$ & 4.32 & 8.5 & $2.21(1.08-4.52)$ & $2.60(1.21-5.58)$ \\
\hline high-risk category & $15 / 460$ & $32 / 523$ & 4.54 & 7.99 & $1.99(1.05-3.76)$ & $1.93(1.02-3.65)$ \\
\hline$p$ for heterogeneity & & & & & 0.90 & 0.89 \\
\hline Low-risk category & $1 / 707$ & $1 / 282$ & 0.17 & 0.43 & $4.02(0.21-75.58)$ & $5.77(0.25-133.23)$ \\
\hline Moderate-/high-risk category & $28 / 1021$ & $49 / 1070$ & 3.71 & 5.85 & $2.04(1.25-3.31)$ & $1.70(1.05-2.76)$ \\
\hline$p$ for heterogeneity & & & & & 0.86 & 0.77 \\
\hline
\end{tabular}

Abbreviations: CI, confidence interval; HR, hazard ratio; sdLDL-C, small dense low-density lipoprotein cholesterol

${ }^{a}$ For the analysis in the overall subjects, age, sex, systolic blood pressure, use of antihypertensive agents, hemoglobin A1c, use of glucose-lowering agents, serum high-density lipoprotein cholesterol, use of lipid-modifying agents, body mass index, estimated glomerular filtration rate, electrocardiogram abnormalities, current smoking, current drinking, and regular exercise were included in the relevant Cox model as covariates.

${ }^{\mathrm{b}}$ For the subgroup analysis of diabetic status, age, sex, systolic blood pressure, use of antihypertensive agents, serum high-density lipoprotein cholesterol, use of lipid-modifying agents, body mass index, estimated glomerular filtration rate, electrocardiogram abnormalities, current smoking, current drinking, and regular exercise were included in the relevant Cox model as covariates.

${ }^{\mathrm{c}} \mathrm{CVD}$-related comorbidities were defined as the presence of diabetes, chronic kidney disease, and/or peripheral artery disease. For the subgroup analysis of the status of CVD-related comorbidities, age, sex, systolic blood pressure, use of antihypertensive agents, serum high-density lipoprotein cholesterol, use of lipid-modifying agents, body mass index, electrocardiogram abnormalities, current smoking, current drinking, and regular exercise were included in the relevant Cox model as covariates.

${ }^{d}$ For the risk assessment status were classified according to the guideline from Japan Atherosclerosis Society. For the subgroup analysis of the risk assessment status, use of antihypertensive agents, use of lipid-modifying agents, body mass index, electrocardiogram abnormalities, current drinking, and regular exercise were included as covariates.

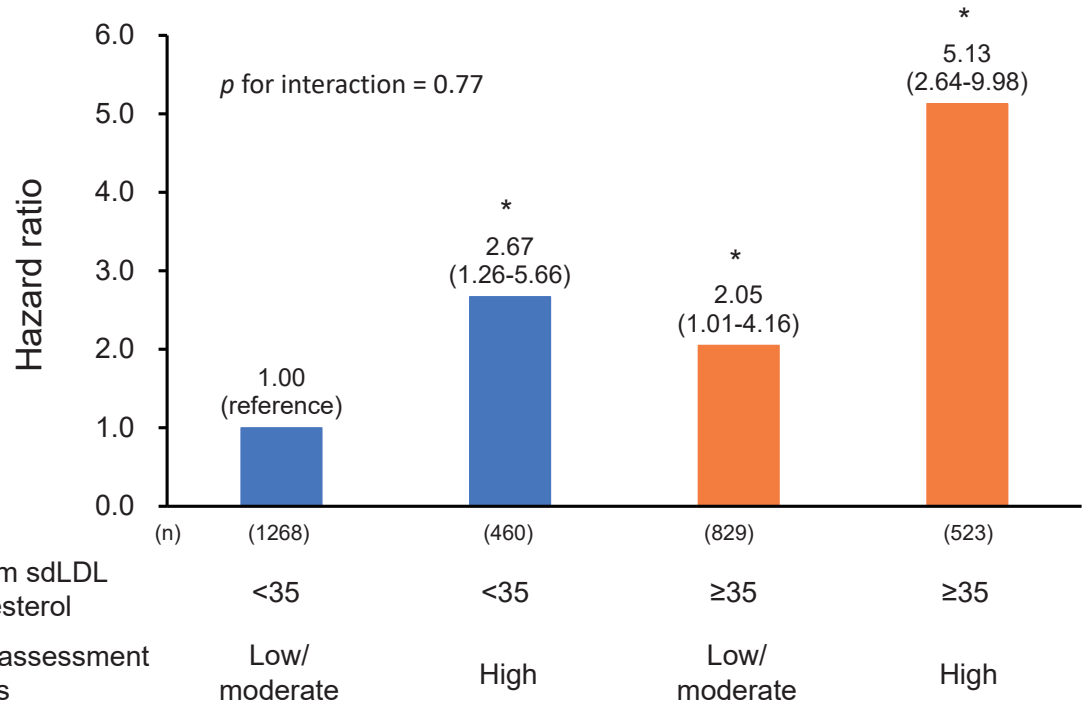

Fig. 2. Multivariable-adjusted hazard ratios for the development of coronary heart disease according to serum small dense low-density lipoprotein cholesterol and the risk assessment status

Abbreviations: sdLDL, small dense lowdensity lipoprotein.

${ }^{*} p<0.05$ vs. reference.

Adjusted for use of antihypertensive agents, use of lipid-modifying agents, body mass index, ECG abnormalities, current drinking, and regular exercise. 
the level of statistical significance in subjects with lower levels of serum LDL and HDL cholesterol (Supplementary Fig.4).

\section{Discussion}

The present study clearly demonstrated that a higher serum sdLDL cholesterol level was associated with increased risk for the development of CHD not only among the participants overall but also among subjects in the high-risk category for CVD, which was defined as recommended in the guidelines of the Japan Atherosclerosis Society. Subjects with higher cardiovascular risk and a higher serum sdLDL cholesterol level had an additively greater risk of CHD events than those with low/moderate cardiovascular risk and a lower serum sdLDL cholesterol level. Moreover, it is noteworthy that the excess risk of a higher serum sdLDL cholesterol level on CHD events was observed among subjects in the high-risk category for CVD regardless of the serum levels of other lipid parameters. These findings provide evidence that the measurement of serum sdLDL cholesterol is clinically valuable for estimating the future onset of $\mathrm{CHD}$ beyond the predefined cardiovascular risks.

There have been a few studies addressing the association between serum sdLDL cholesterol and CHD risk in the high-risk population for CVD. In the Multi-Ethnic Study of Atherosclerosis (MESA), a higher serum sdLDL cholesterol level was associated with the development of CHD among overall study participants, but the association was not statistically significant among subjects with type 2 diabetes ${ }^{11)}$. Our result was inconsistent with this finding. The reasons for this discrepancy are not entirely clear, but it may be related to the accuracy of the follow-up data or statistical power of the MESA study, as the MESA study failed to reveal a significant association between serum LDL cholesterol and CHD, even though serum LDL cholesterol is an established risk factor for CHD in patients with diabetes ${ }^{19)}$.

The strengths of the present study were its longitudinal population-based design, almost perfect follow-up of subjects, and accuracy in the diagnosis of $\mathrm{CHD}$ on the basis of medical records and autopsy findings. However, some potential limitations of this study should be noted. First, we were able to measure the serum sdLDL cholesterol level and other risk factors only once at baseline. We were also unable to obtain information about medical treatments during the follow-up period. In addition, information on the family history of early onset of CHD was not available, likely leading to an underestimation of the absolute risk based on the Suita score. This limitation could lead to misclassification of these variables, which in turn would weaken the association found in the current study, biasing the results toward the null hypothesis. Second, we could not conduct the subgroup analyses among subjects with or without chronic kidney disease or peripheral artery disease, because of the limited number of subjects with either disease. Third, we measured serum sdLDL cholesterol levels using frozen serum samples stored at $-80^{\circ} \mathrm{C}$ for seven years, while serum sdLDL cholesterol levels were unlikely to be affected by the frozen storage ${ }^{20)}$. Finally, since these analyses were conducted only in one cohort of Japanese, the generalizability of our findings might be limited. Therefore, our findings should be validated in other cohorts of various ethnic populations.

\section{Conclusions}

Elevated sdLDL cholesterol level was associated with the development of CHD among subjects at high-risk category as estimated according to the guidelines of the Japan Atherosclerosis Society. High-risk approaches for preventing CHD using serum sdLDL cholesterol measurement adhering with the existing guideline are likely to provide additional information against the burden of CHD in Japan. Further investigations are required to clarify whether serum sdLDL cholesterol would be an interventional target for reducing the risk of $\mathrm{CHD}$ events among subjects at high cardiovascular risk.

\section{Acknowledgements and Notice of Grant Support}

We would like to gratefully and sincerely thank Professor Yoshinao Oda, Professor Toru Iwaki, and their colleagues at the Department of Anatomic Pathology and Department of Neuropathology, Graduate School of Medical Sciences, Kyushu University, who provided expertise and insight into the autopsy findings that greatly assisted our research. The statistical analyses were carried out using the computer resources offered under the category of General Projects by the Research Institute for Information Technology, Kyushu University.

This study was supported in part by Grants-inAid for Scientific Research (A) (JP16H02692) and (B) (JP16H05850), JP17H04126, and JP18H02737) and (C) (JP17K09114, JP17K09113, JP17K01853, JP18K07565, JP18K09412, and JP19K07890) and (Early-Career Scientists) (JP18K17925, and JP18K17382) from the Ministry of Education, Culture, Sports, Science and Technology of Japan; by 
Health and Labour Sciences Research Grants of the Ministry of Health, Labour and Welfare of Japan (H29-Junkankitou-Ippan-003, and H30-Shokuhin[Sitei]-005); and by the Japan Agency for Medical Research and Development (JP19dk0207025, JP19ek0210082, JP19ek0210083, JP19km0405202, JP19ek0210080, JP19fk0108075). In addition, this study was sponsored by DENKA SEIKEN Co., Ltd. (Tokyo, Japan). The sponsor of the study had no role in the study design, conduct of the study, data collection, data interpretation or preparation of the report.

\section{Conflict of Interest}

Toshiharu Ninomiya received research funding from DENKA SEIKEN Co., Ltd. The other authors declare that they have no conflicts of interest.

\section{References}

1) Collaborators GBDCoD: Global, regional, and national age-sex-specific mortality for 282 causes of death in 195 countries and territories, 1980-2017: a systematic analysis for the Global Burden of Disease Study 2017. Lancet, 2018; 392: 1736-1788

2) Vital Health and Social Statistics Office, Ministry of Health, Labour and Welfare: Vital Statistics. Available from:https://www.e-stat.go.jp/dbview?sid=0003214721, Accessed 2020 Jan 17

3) Baigent C, Keech A, Kearney PM, Blackwell L, Buck G, Pollicino C, Kirby A, Sourjina T, Peto R, Collins R, Simes R; Cholesterol Treatment Trialists' (CTT) Collaborators: Efficacy and safety of cholesterol-lowering treatment: prospective meta-analysis of data from 90,056 participants in 14 randomised trials of statins. Lancet, 2005; 366: 12671278

4) Kinoshita M, Yokote $K$, Arai H, Iida M, Ishigaki Y, Ishibashi S, Umemoto S, Egusa G, Ohmura H, Okamura T, Kihara S, Koba S, Saito I, Shoji T, Daida H, Tsukamoto K, Deguchi J, Dohi S, Dobashi K, Hamaguchi H, Hara M, Hiro T, Biro S, Fujioka Y, Maruyama C, Miyamoto Y, Murakami Y, Yokode M, Yoshida H, Rakugi H, Wakatsuki A, Yamashita S; Committee for Epidemiology and Clinical Management of Atherosclerosis: Japan Atherosclerosis Society (JAS) Guidelines for Prevention of Atherosclerotic Cardiovascular Diseases 2017. J Atheroscler Thromb, 2018; 25: 846-984

5) Nishimura K, Okamura T, Watanabe M, Nakai M, Takegami M, Higashiyama A, Kokubo Y, Okayama A, Miyamoto Y: Predicting coronary heart disease using risk factor categories for a Japanese urban population, and comparison with the framingham risk score: the suita study. J Atheroscler Thromb, 2014; 21: 784-798

6) Galeano NF, Al-Haideri M, Keyserman F, Rumsey SC, and Deckelbaum RJ: Small dense low density lipoprotein has increased affinity for LDL receptor-independent cell surface binding sites: a potential mechanism for increased atherogenicity. J Lipid Res, 1998; 39: 1263-1273

7) Anber V, Griffin BA, McConnell M, Packard CJ, and
Shepherd J: Influence of plasma lipid and LDL-subfraction profile on the interaction between low density lipoprotein with human arterial wall proteoglycans. Atherosclerosis, 1996; 124: 261-271

8) Tribble DL, Rizzo M, Chait A, Lewis DM, Blanche PJ, and Krauss RM: Enhanced oxidative susceptibility and reduced antioxidant content of metabolic precursors of small, dense low-density lipoproteins. Am J Med, 2001; 110: 103-110

9) Arai H, Kokubo Y, Watanabe M, Sawamura T, Ito Y, Minagawa A, Okamura T, Miyamato Y: Small dense lowdensity lipoproteins cholesterol can predict incident cardiovascular disease in an urban Japanese cohort: the Suita study. J Atheroscler Thromb, 2013; 20: 195-203

10) Hoogeveen RC, Gaubatz JW, Sun W, Dodge RC, Crosby JR, Jiang J, Couper D, Virani SS, Kathiresan S, Boerwinkle E, Ballantyne CM: Small dense low-density lipoprotein-cholesterol concentrations predict risk for coronary heart disease: the Atherosclerosis Risk In Communities (ARIC) study. Arterioscler Thromb Vasc Biol, 2014; 34: 1069-1077

11) Tsai MY, Steffen BT, Guan W, McClelland RL, Warnick R, McConnell J, Hoefner DM, Remaley AT: New automated assay of small dense low-density lipoprotein cholesterol identifies risk of coronary heart disease: the Multiethnic Study of Atherosclerosis. Arterioscler Thromb Vasc Biol, 2014; 34: 196-201

12) Higashioka M, Sakata S, Honda T, Hata J, Yoshida D, Hirakawa Y, Shibata M, Goto K, Kitazono T, Osawa H, Ninomiya T: Small dense low-density lipoprotein cholesterol and the risk of coronary heart disease in a Japanese community. J Atheroscler Thromb, 2020; 27: 669-682

13) Hata J, Ninomiya T, Hirakawa Y, Nagata M, Mukai N, Gotoh S, Fukuhara M, Ikeda F, Shikata K, Yoshida D, Yonemoto K, Kamouchi M, Kitazono T, Kiyohara Y: Secular trends in cardiovascular disease and its risk factors in Japanese: half-century data from the Hisayama Study (1961-2009). Circulation, 2013; 128: 1198-1205

14) Ninomiya T: Japanese Legacy Cohort Studies: The Hisayama Study. J Epidemiol, 2018; 28: 444-451

15) Ito Y, Fujimura M, Ohta $M$ and Hirano T: Development of a homogeneous assay for measurement of small dense LDL cholesterol. Clin Chem, 2011; 57: 57-65

16) Thygesen K, Alpert JS, White HD; Joint ESC/ACCF/ AHA/WHF Task Force for the Redefinition of Myocardial Infarction: Universal definition of myocardial infarction. Eur Heart J, 2007; 28: 2525-2538

17) Horio M, Imai E, Yasuda $Y$, Watanabe $T$ and Matsuo $S$ : Modification of the CKD epidemiology collaboration (CKD-EPI) equation for Japanese: accuracy and use for population estimates. Am J Kidney Dis, 2010; 56: 32-38

18) Group KDIGOKCW: KDIGO 2012 clinical practice guideline for the evaluation and management of chronic kidney disease. Kidney Int Suppl, 2013; 3: 1-150

19) Huang ES, Meigs JB and Singer DE: The effect of interventions to prevent cardiovascular disease in patients with type 2 diabetes mellitus. Am J Med, 2001; 111: 633-642

20) Hirano T, Ito $Y$, Saegusa $H$ and Yoshino G: A novel and simple method for quantification of small, dense LDL. J Lipid Res, 2003; 44: 2193-2201 


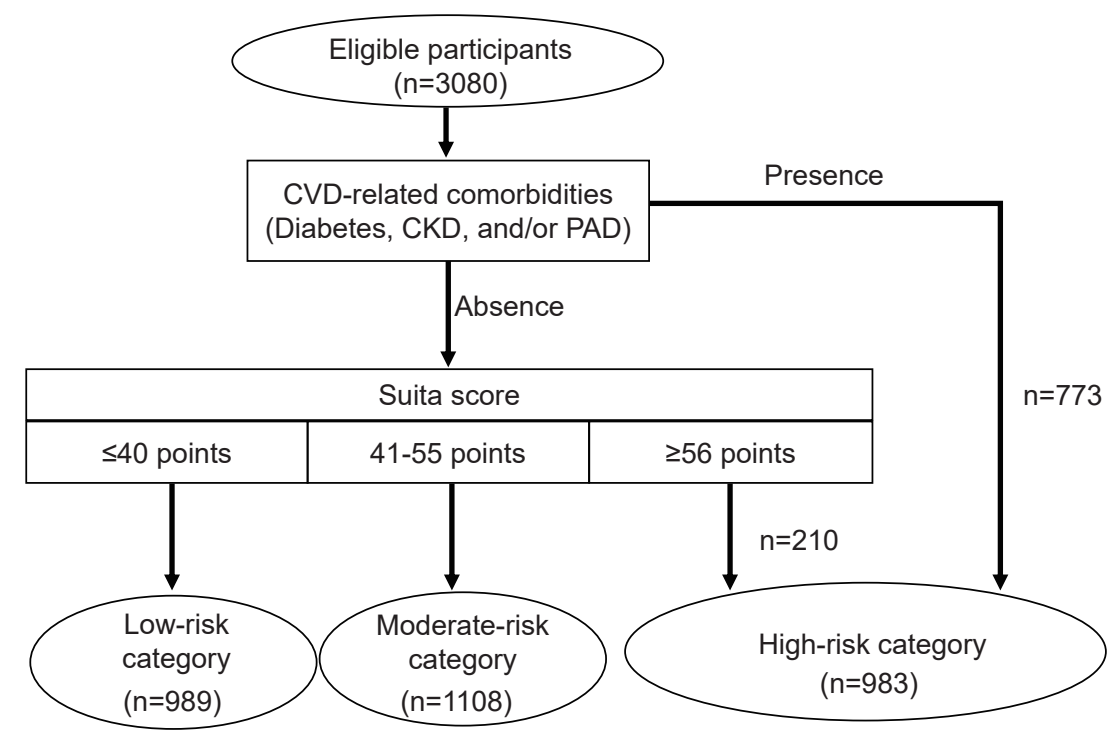

Supplementary Fig. 1. Flowchart of the risk assessment according to the guidelines from the Japan Atherosclerosis Society

Abbreviations: CKD, chronic kidney disease; CVD, cardiovascular disease; PAD, peripheral artery disease.

Suita score was calculated on the basis of the following risk factors at baseline for each participant: age, sex, smoking habits, blood pressure level, serum high-density lipoprotein cholesterol, serum low-density lipoprotein cholesterol, and prediabetes among subjects without CVDrelated comorbidities. Eligible participants $(n=3,080)$ were selected from 3,384 study population, after excluding 8 subjects who did not consent to participate in the study, 223 subjects who had past history of CVD, and 73 subjects for whom serum sdLDL cholesterol data were lacking.

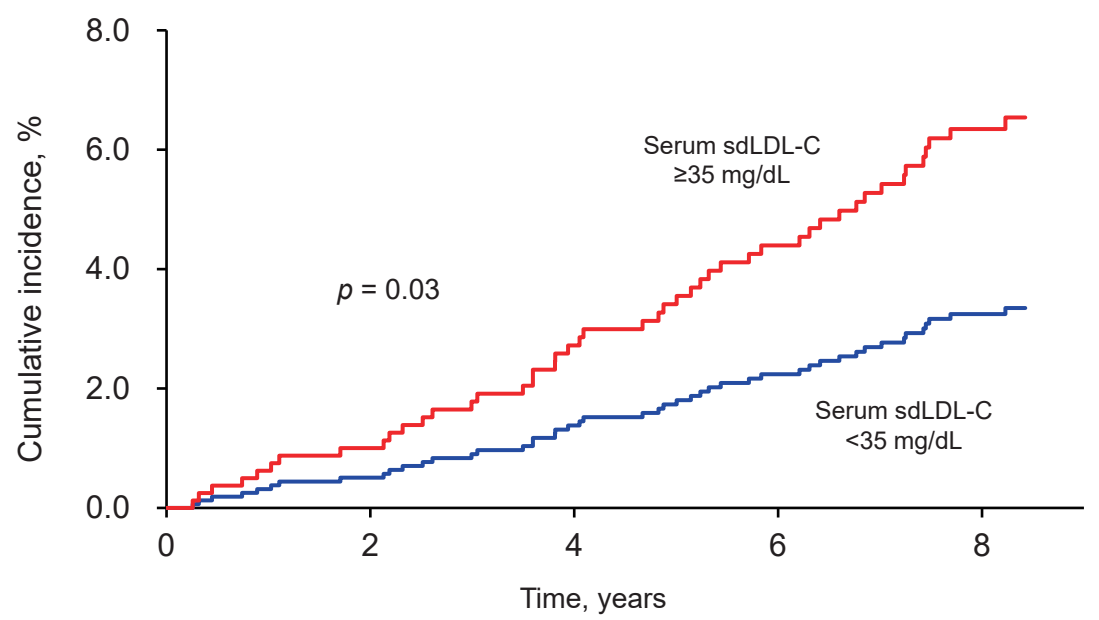

Supplementary Fig. 2. Age- and sex-adjusted cumulative incidence of coronary heart disease according to serum small dense lowdensity lipoprotein cholesterol among subjects in the high-risk category $(n=983)$

Abbreviations: sdLDL-C, small dense low-density lipoprotein cholesterol. 
(a)

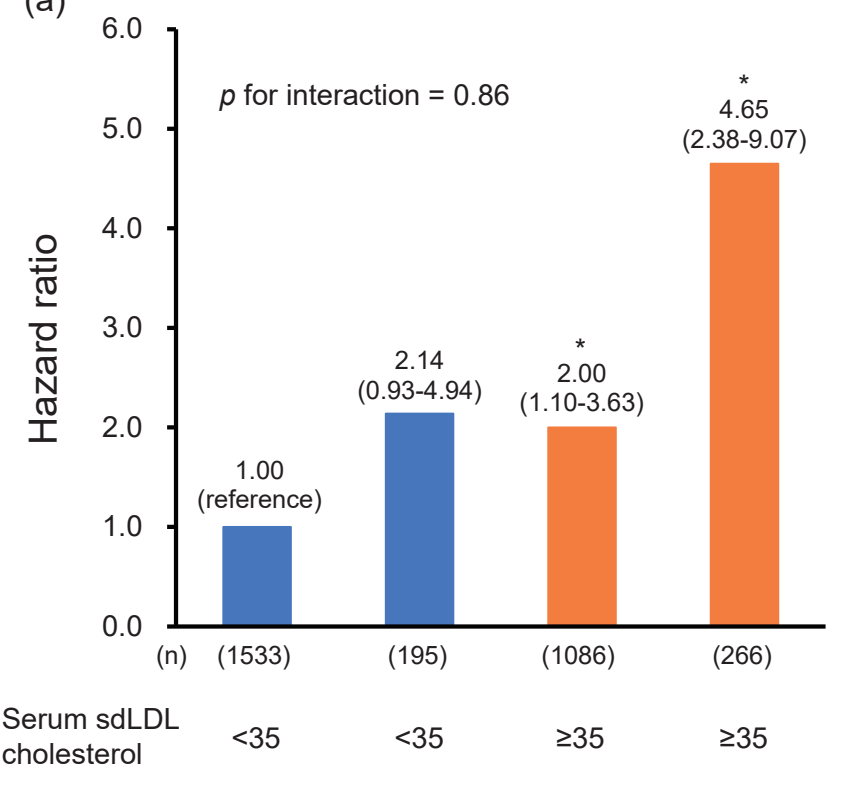

Diabetes
$(-)$ (b)

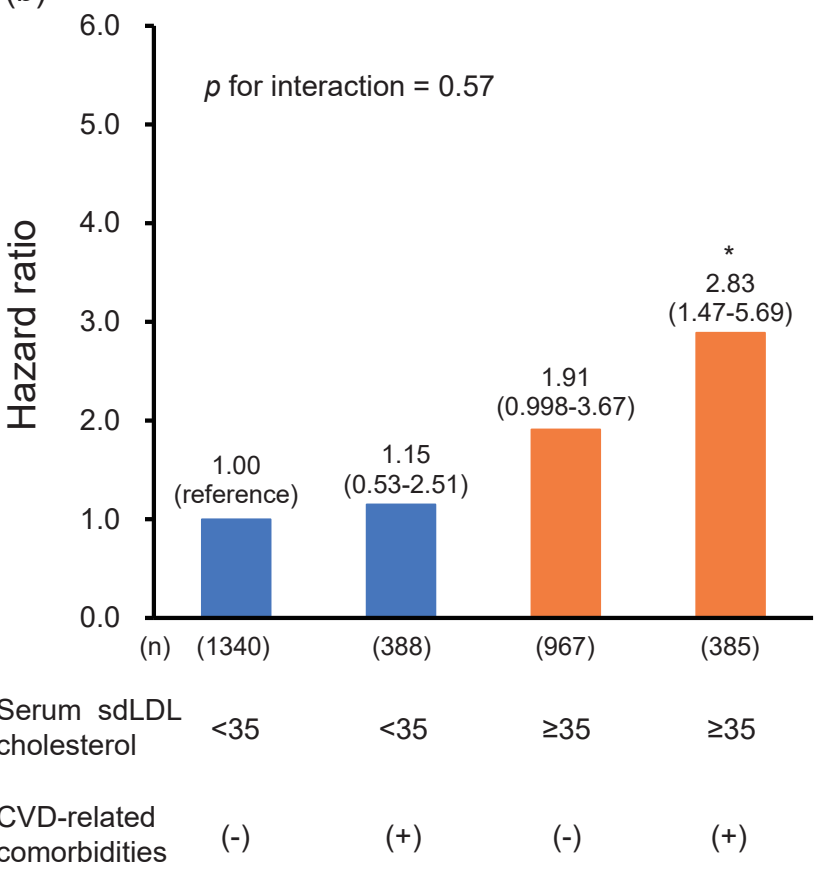

Supplementary Fig.3. Multivariable-adjusted hazard ratios for the development of coronary heart disease according to serum small dense low-density lipoprotein cholesterol and presence or absence of (a) diabetes or (b) CVD-related comorbidities

Abbreviations: CVD, cardiovascular disease; sdLDL, small dense low-density lipoprotein.

${ }_{p}<0.05$ vs. reference.

Adjusted for age, sex, systolic blood pressure, use of antihypertensive agents, high-density lipoprotein cholesterol, use of lipid-modifying agents, body mass index, estimated glomerular filtration rate, electrocardiogram abnormalities, current smoking, current drinking, and regular exercise. In the analysis for (b), the estimated glomerular filtration rate was excluded from the adjusted factors.

Supplementary Table 1. Correlation between serum small dense low-density lipoprotein cholesterol level and other lipid parameters among subjects in the high-risk category

\begin{tabular}{lccc}
\hline Parameter & $n$ & $\begin{array}{c}\text { Spearman's rank correlation } \\
\text { coefficient }\end{array}$ & $p$ value \\
\hline Serum LDL cholesterol & 983 & 0.65 & $<0.001$ \\
Serum non-HDL cholesterol & 983 & 0.76 & $<0.001$ \\
Serum HDL cholesterol & 983 & -0.30 & $<0.001$ \\
Serum triglycerides & $974^{\mathrm{a}}$ & 0.62 & $<0.001$ \\
\hline
\end{tabular}

Abbreviations: HDL, high-density lipoprotein; LDL, low-density lipoprotein.

${ }^{a}$ Only subjects with available fasting blood samples were included in the analysis using serum triglycerides 

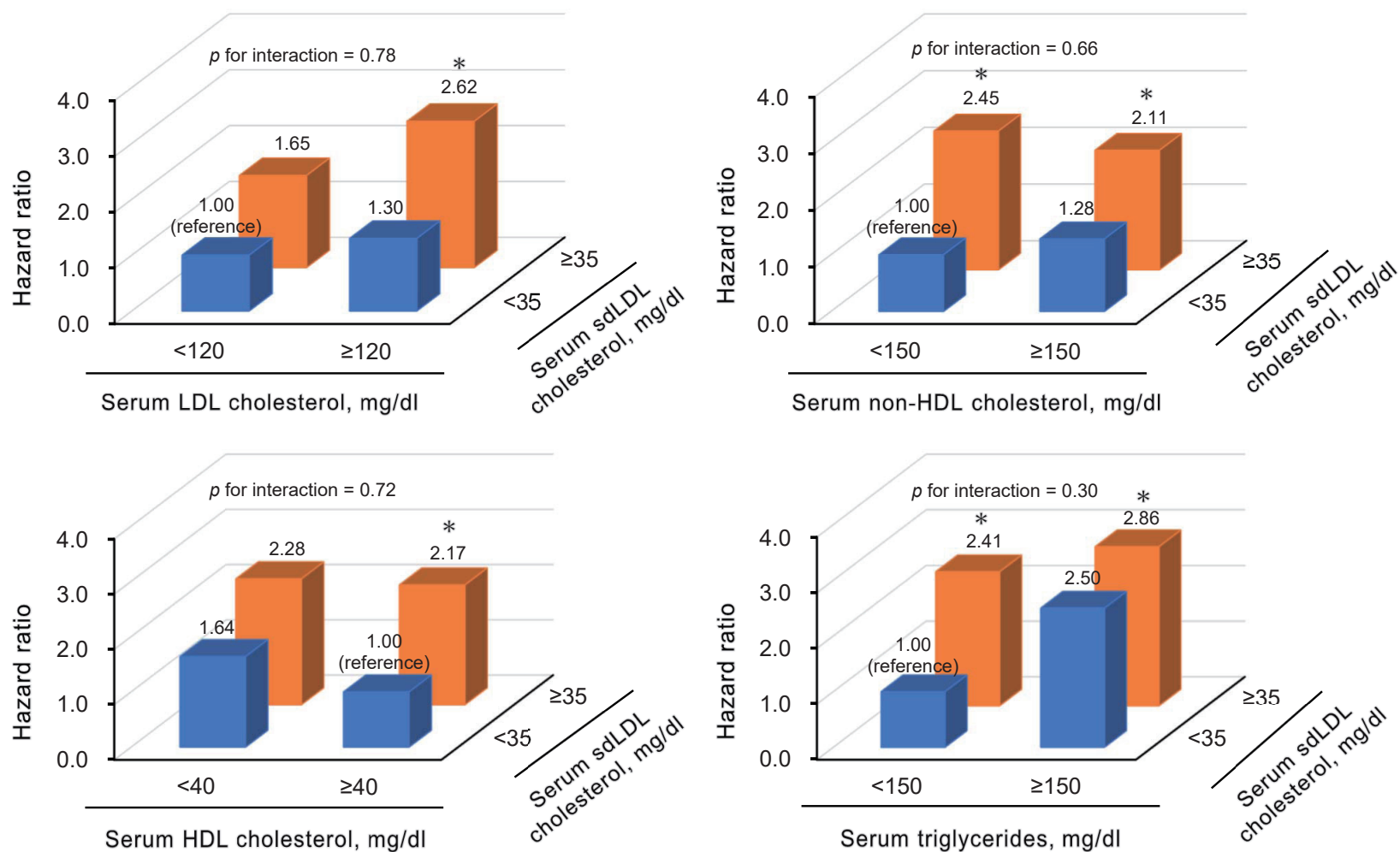

Supplementary Fig.4. Multivariable-adjusted hazard ratios for the development of coronary heart disease according to serum sdLDL cholesterol and other lipid parameters among subjects in the high-risk category $(n=983)$

Abbreviations: HDL, high-density lipoprotein; LDL, low-density lipoprotein; sdLDL, small dense low-density lipoprotein.

${ }^{*} p<0.05$ vs. reference.

Hazard ratios were adjusted for age, sex, systolic blood pressure, antihypertensive drugs, hemoglobin A1c, antidiabetic medication, HDL cholesterol, lipidlowering drugs, body mass index, estimated glomerular filtration rate, electrocardiogram abnormalities, current smoking, current drinking, and regular exercise.

In the analysis of subgroups of serum HDL cholesterol, serum HDL cholesterol was excluded from the adjusted factors.

In the analysis of serum triglycerides, only subjects with available fasting blood samples were included in the analysis using serum triglyceride $(n=974)$. 